\title{
Expert Roundtable Discussion on Penile Prosthesis Infection Prevention Measures
}

\author{
Eric Chung, $, 11,2$ Martin S. Gross, ${ }^{3}$ Koenraad van Renterghem, ${ }^{4}$ Jay Simhan ${ }^{5}$ \\ ${ }^{1}$ University of Queensland, Princess Alexandra Hospital, Brisbane, Australia ${ }^{2}$ AndroUrology Centre, Brisbane and Sydney, Australia ${ }^{3}$ Dartmouth-Hitchcock Medical \\ Center, Lebanon, United States ${ }^{4}$ Department of Urology, Jessa Hospital and Hasselt University, Hasselt, Belgium ${ }^{5}$ Einstein Medical Center, Philadelphia, United States
}

Inflatable penile prosthesis (IPP) implantation remains the standard of care for men with medical-refractory erectile dysfunction. However, IPP infection remains one of the most consequential complications of penile prosthesis surgery[1,2]. Apart from the patient morbidity and explant of the infected device, infection can also be associated with significant corporal fibrosis with ensuing reduction in penile size and more difficult re-implantation, as well as adverse psychosexual and socioeconomic effects[3,4]. The incidence of IPP infection has decreased over the last decade because of better surgical care pathways coupled with antibiotic-coated prostheses and surgical technique improvements[5].

IPP infection should be differentiated from infection of the surgical site alone[4]. Most implant infections usually occur within the first 6 weeks because of bacterial contamination at the time of surgery. In contrast, chronic infections or infections occurring after 6 weeks are often subclinical, with patients presenting with chronic pain around the device components or device extrusion[6].

The following commentary is a summary of the experts' round table discussion by key opinion leaders and high-volume surgeons from North America, Europe, and Asia Pacific regions at the recent Sociéte Internationale d'Urologie (SIU) Academy educational webinar. Contemporary literature and clinical evidence regarding the prevention and management of penile implant infection were discussed[2,6,7]. A detailed analysis of all relevant studies, including a full surgical description, is not feasible in this commentary.

\section{Primary Penile Implant Infection}

Modifications of preoperative and perioperative risk factors with appropriate use of antibiotic coverage and modern IPP have significantly reduced infection rates over the years. Pre-existing medical comorbidities such as diabetes, smoking status, immunosuppression, radiation history, neurological disorder, previous pelvic surgery, older age, and obesity have all been shown to increase the risk of peri-prosthetic infection[2-4,6-9], and optimisation of these risk factors might mitigate IPP infection. All patients should undergo routine preoperative urine microscopy tests and skin checks. Appropriate precautions to minimize contamination by common skin organisms include a preoperative shower with an antibacterial agent, intraoperative hair removal and use of alcoholic formulations for skin preparation[6,7]. Perioperative antibiotic prophylaxis, ideally given at least 1 hour before surgery, and the use of antibiotic-coated devices have been well documented to minimize intraoperative infection risk[2,6,7]. While the nature of intravenous antibiotics is likely dependent on local institution antibiotics policy, the combination of antibiotics covering both gram-positive and gram-negative bacteria is preferred. The co-administration of an antifungal is usually reserved for patients with obesity or poorly controlled diabetes, or in certain geographical locations, or in the setting of salvaging an infected IPP $[2,3]$. The group consensus recommendation to augment coverage with antifungals is in contradistinction to existing antimicrobial recommendations of the American Urological Association and the European Association of Urology but is felt to be necessary given contemporary studies that have demonstrated the prevalence of fungal organisms in these high-risk penile implant populations.

\section{Key Words}

Penile prosthesis, infection, erectile dysfunction, salvage surgery, revision prosthesis

\section{Competing Interests}

None declared

\section{Article Information}

Received on August 8, 2021

Accepted on August 27, 2021

Soc Int Urol J.2021;2(6):380-381

DOI: https://doi:10.48083/GVCC5010 
Standard surgical protocols such as appropriate full protective surgical attire and limiting the number of staff and traffic within the operating room should be instituted[7]. Attention to surgical wound sterility with a small surgical field, the use of antibiotic solution irrigation and minimizing device-skin contact, have been described to improve surgical sterility[6,7]. There is currently no evidence to suggest that a particular surgical approach has a higher infection risk[1].

Postoperative antimicrobial prophylaxis is often given especially in high-risk groups (eg, people with diabetes) although this remains a surgeon preference. Patients are advised to maintain high standards of hygiene and refrain from sexual activity in the first 4 to 6 weeks after surgery. Any signs of impending infection such as fever or early cellulitis should be brought to the attention of the surgeon immediately.

\section{Revision Penile Implant Infection}

Patients with pre-existing risk factors will always have a higher risk of IPP infection after revision surgery compared with after primary surgery $[3,6]$. Those operated on for impending device component erosion are at increased risk of infection, especially if the revision surgery requires additional or complex procedures such as graft reconstruction[3].

In revision surgery, aggressive irrigation of all surgical sites and excision of pseudo-capsule may remove bacterial seeding within the biofilm, which is often responsible for delayed device infection $[2,4,7]$. Any device that is older than 5 years should ideally be exchanged at the revision surgery. The existing reservoir can be retained in the absence of infection or eroded penile prosthetic component if the defunctionalized reservoir is located within the extraperitoneal and retropubic space $[6,7]$,

\section{References}

1. Levine LA, Becher E, Bella A, Brant WO, Kohler TS, MartinezSalamanca JI. Penile prosthesis surgery: current recommendations from the International Consultation on Sexual Medicine. J Sex Med.2016;13:489-518. D0I: 10.1016/j.jsxm.2016.01.017

2. Swanton AR, Munarriz RM, Gross MS. Updates in penile prosthesis infections. Asian J Androl.2020;22(1):28-33.

3. Chung E. Penile prosthesis implant in the special populations: diabetics, neurogenic conditions, fibrotic cases, concurrent urinary incontinence, and salvage implants. Asian J Androl.2020;22(1):39-44.

4. Al-Shaiji TF, Yaiesh SM, Al-Terki AE, Alhajeri FM. Infected penile prosthesis: literature review highlighting the status quo of prevention and management. Aging Male.2020;23(5):447-456.

5. Chung E. Penile prosthesis implant: Scientific advances and technological innovations over the last four decades. Trans/ Androl Urol.2017;6(1):37-45. but needs to be emptied and capped to prevent fluid accumulation and subsequent risk of infection[1]. Many surgeons will prescribe postoperative antibiotics for patients in revision and salvage cases.

\section{Salvage Penile Implant Infection}

In the early stage of IPP infection, a trial course of intravenous antibiotics with or without antifungal may be appropriate in the absence of systemic sepsis or purulent discharge[1,2]. However, the decision for surgical intervention should be made if there is a progression of sepsis or evidence of device extrusion $[1,3,4]$. The decision to conserve an IPP or not depends on the patient's presentation and clinical status, the timeline and onset of infection, and response to the antibiotic treatment [10].

While explant of the infected device without salvage remains the safest option, salvage protocol with revision penile prosthesis implant can be carried out if there is an absence of tissue necrosis and purulence in the corporal bodies or scrotum[1-3,7]. In the salvage cases, all components of the device should be removed with aggressive mechanical lavage based on various versions of Mulcahy's washout protocols using a series of antiseptic solutions consisting of half-strength povidone-iodine, half-strength hydrogen peroxide, and combination antibiotic agents. An immediate device replacement importantly preserves sexual function and penile length and further prevents corporal fibrosis. A malleable implant is a simpler and cheaper alternative to IPP that can be used at the time of salvage surgery and facilitate eventual conversion to inflatable implant in the near or long-term future[1]. For a delayed re-implantation, use of a vacuum erection device may help in obtaining good results and reducing fibrosis and the shortening of the penis.

6. Hebert KJ, Kohler TS. Penile prosthesis infection: myths and realities. World J Mens Health.2019;37(3):276-87

7. Best JC, Clavijo RI. Best practices for infection prevention in penile prosthesis surgery. Curr Opin Urol.2020;30(3):302-308.

8. Gon LM, de Campos CCC, Voris BR, Passeri LA, Fregonesi A, Zanettini Riccetto CL. A systematic review of penile prosthesis infection and meta-analysis of diabetes role. BMC Urol.2021;21:35. DOI: https://doi.org/10.1186/s12894-020-00730-2

9. Carrasquillo RJ, Munarriz RM, Gross MS. Infection prevention considerations for complex penile prosthesis recipients. Curr Urol Rep.2019;20(3):12

10. Barlotta R, Foote C, Simhan J. Penile prosthesis salvage: Review of past and current practices. Curr Sex Health Rep.2019;11:185-189. 